\title{
Sonografie der normalen Gallenwege
}

Thomas Müller

Der perkutane Ultraschall führt bei Verdacht auf Erkrankungen der Gallenwege zu einer schnellen Diagnose [1]. Die richtigen Strukturen zu erkennen, ist jedoch nicht immer einfach: Vor allem der dünne Ductus hepatocholedochus (DHC) ist nur schwer zu finden. Der Beitrag gibt Tipps, die Ihnen die Suche erleichtern. Außerdem erfahren Sie, woran Sie erkennen, ob der DHC pathologisch verändert ist - oder nicht.

\section{Vor dem Start}

Indikationen I Die Sonografie der Gallenwege ist sinnvoll bei Patienten mit:

- (rechtsseitigen, wiederkehrenden) Oberbauchschmerzen

- Ikterus

- einer Erhöhung der Cholestase anzeigenden Laborparameter (GPT, rGT, AP, Bilirubin)

- einer akuten Pankreatitis

Im B-Bild zeigen sich flüssigkeitsgefüllte Gänge und Gefäße als echofreie tubuläre Strukturen. Kommt es durch Steine oder Tumore zu einer Obstruktion der Gänge, kann man dies durch die Sonografie erkennen und die weitere Diagnostik und Therapie planen.

Technische Voraussetzungen | Für die Untersuchung genügt eine normale curved-array-Abdominalsonde $(2,5-5 \mathrm{MHz})$. Optional ist der Farbdoppler.

Patientenvorbereitung | Eine Nahrungskarenz ist nur selten erforderlich. Wurden zuvor gashaltige Getränke eingenommen, kann dies die Untersuchung jedoch erschweren.

Lagerung I Der Patient befindet sich in Rückenlage oder dreht sich gegebenenfalls in die Linksseitenlage.

\section{Sonografie der Gallenwege: So wird's gemacht}

Lokalisation der intrahepatischen Gallenwege I Die intrahepatischen Gallenwege werden im Rahmen der systematischen Untersuchung des Leberparenchyms mit beurteilt. Sie laufen parallel zu den Pfortaderästen und sind in der gesunden Leber so zart, dass sie kaum zu sehen sind. Leberzentral kann man sie aber bei jungen schlanken Menschen mitunter als schmale echofreie tubuläre Strukturen erkennen ( Abb. 1). Im Umkehrschluss bedeutet das: Deutlich sichtbare Gallenwege neben den Pfortaderästen in der Peripherie sind ein Hinweis auf einen gestörten Gallenabfluss.

Lokalisation der extrahepatischen Gallenwege I Der Ductus hepatocholedochus (DHC) läuft aus der Leberpforte parallel der Pfortader (Vena portae, VP) zum Pankreaskopf und durch diesen zur Papille im Duodenum ( $\triangleright$ Abb. 2). Mit der Pfortader kreuzt er die untere Hohlvene (Vena cava inferior, VCI) in spitzem Winkel. Auch im Ligamentum hepatoduodenale, aber meist schräg zum DHC, läuft die Arteria hepatica propria $(\mathrm{AH})$.

Für die Schallkopfposition heißt das: Sie denken sich eine Linie vom Nabel des Patienten zu seinem rechten Humeruskopf und setzen unterhalb des Rippenbogens Ihren Schallkopf auf diese Linie ( $\triangle$ Abb. 3). Hier suchen Sie jetzt parallel laufende echofreie tubuläre Strukturen. Schallkopf-fern, also in der Tiefe, liegt die VCI. Ventral davon („darüber") liegt die Pfortader.

Die normale VCI weist einen Doppelschlag auf und ihre Weite ist atemmoduliert. Sie finden sie leichter, wenn Sie den Patienten pressen lassen („Valsalva-Manöver“). Gallenwege (Pfeil) stellen sich zentral als schmale echofreie Bänder parallel zu den Pfortaderästen (darunter) dar.

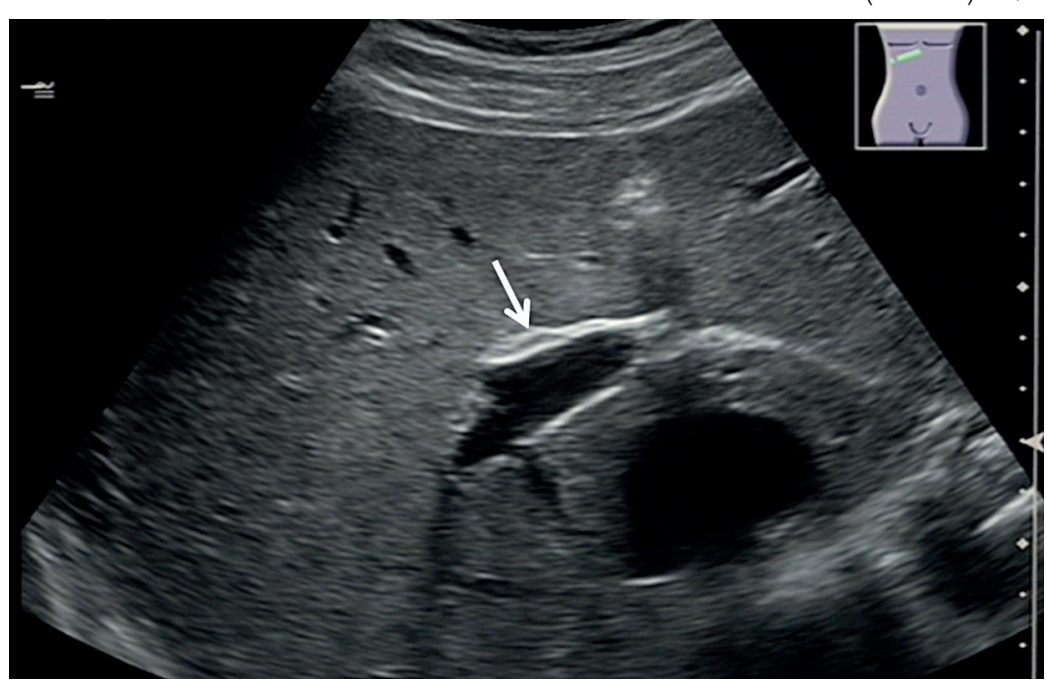




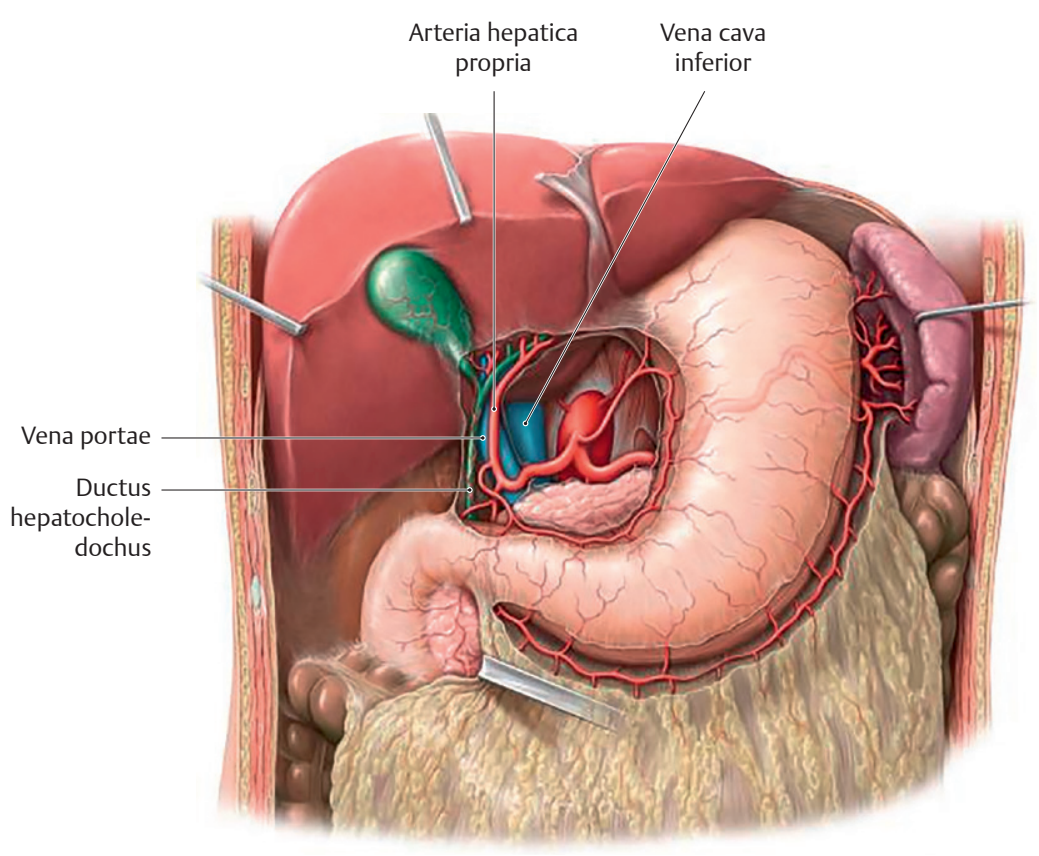

Abb. 2 Bei hochgeklappter Leber sieht man hier die Gefäße der Leberpforte: Ductus hepatocholedochus, Arteria hepatica propria und Vena portae, darunter in der Tiefe die Vena cava inferior.

Quelle: Schünke M, Schulte E, Schumacher E. Prometheus. LernAtlas der Anatomie. Innere Organe. Illustration von K. Wesker. 2. Auflage Stuttgart: Thieme 2009.
Auffinden des DHC I Ventral der Pfortader, also am nächsten am Schallkopf, und parallel zu ihr liegt der DHC $(\checkmark$ Abb. 4). Im gesunden Zustand ist er meist die dünnste dieser drei Strukturen und entsprechend schwierig zu finden. Die Schichtung von dorsal nach ventral ist aber immer gleich. Der Verlauf der Arteria hepatica propria ist variabel, sie liegt meist dorsal des DHC, gelegentlich auch ventral, aber nur selten langstreckig parallel zu ihm.

Cave Pfortader und Arteria hepatica propria können mit dem DHC verwechselt werden.

Die Farbdopplersonografie kann bei der Orientierung helfen:

- Wird die mittlere Geschwindigkeit auf etwa $10 \mathrm{~cm} / \mathrm{s}$ eingestellt und

- ist der Winkel zwischen Schallstrahl und Gefäßen nicht gerade $90^{\circ}$,

stellen sich die Blutgefäße (VCI, VP, AH) farbbelegt dar - der DHC nicht. Gallefluss ist mit der Farbdopplersonografie nicht darstellbar. Diese „Ausschlussdiagnostik“" wird auch von erfahrenen Untersuchern angewandt.

Darstellung der extrahepatischen Gallengänge I Haben Sie den DHC identifiziert, verschieben Sie den Schallkopf in Längsachse zunächst leberwärts (also nach rechts oben), bis Sie die Hepaticusgabel erreichen. Durch leichtes seitliches „Fächern“ mit dem Schallstrahl lässt sich selten auch der winzige Ductus cysticus darstellen.

Dann folgt die gleiche Längsbewegung nach medial unten, Richtung Pankreas. Hier ist zwischen Pankreas und Bauchwand das Duodenum interponiert und Duodenalluft kann die Sicht auf den DHC „versperren“.
Wenn Duodenalluft stört | Sollten Sie wegen der Duodenalluft den DHC nicht erkennen, können Sie

- dosiert komprimieren (und das auch ankündigen!),

- den Patienten bitten, den Bauch vorzuwölben,

- den Patienten bitten, sich auf die linke Seite zu legen und dieselbe Schallkopfposition wieder aufsuchen.

Mit Kompression kann man das Duodenum zusammenpressen, die Luft weicht zur Seite und der DHC wird wieder sichtbar. Beim Herausstrecken des Bauches schiebt das Zwerchfell die Leber nach kaudal und oft wird die Sicht besser. Die Untersuchung in Linksseitenlage gehört grundsätzlich zur Sonografie der Gallenwege. Auch hier „fällt die Leber ins Abdomen“ ( $\downarrow$ Abb. 5).

Oberbauchquerschnitt | Ein weiterer Zugang zum distalen Abschnitt des DHC ist der Oberbauchquerschnitt durch das Pankreas. Der DHC durchzieht den Pankreaskopf von cranial rechts leicht bogenförmig wieder nach rechts zur Papille - er sollte also im Oberbauchquerschnitt im Pankreaskopf auch quer getroffen werden. $\mathrm{Zu}$ sehen ist ein nahe der Duodenalwand gelegener runder echofreier Querschnitt ( Abb. 6). Hier kann man insbesondere papillennahe Steine oder Tumorstenosen erkennen.

\section{Was ist normal?}

Schmal mit erkennbarer Wand | Der gesunde DHC ist schmal und hat eine erkennbare Wand. Diese ist nicht geschichtet, sondern stark echogen. Über die Frage, wie schmal er sein darf, um noch als normal zu gelten, wird derzeit gestritten. Die vielfältigen „Normwerte“ hängen von unterschiedlichen Kollektiven, Messpunkten, der Messung mit oder ohne Wand und einigem mehr ab.

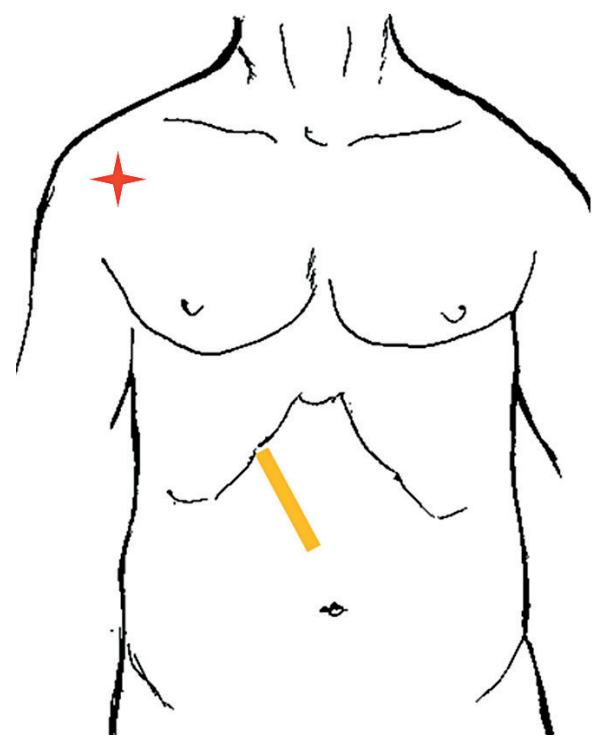

Abb. 3 Der Schallkopf wird auf einer gedachten Linie vom Nabel zum rechten Humeruskopf (roter Stern) des Patienten unterhalb des Rippenbogens platziert (gelbe Linie). 


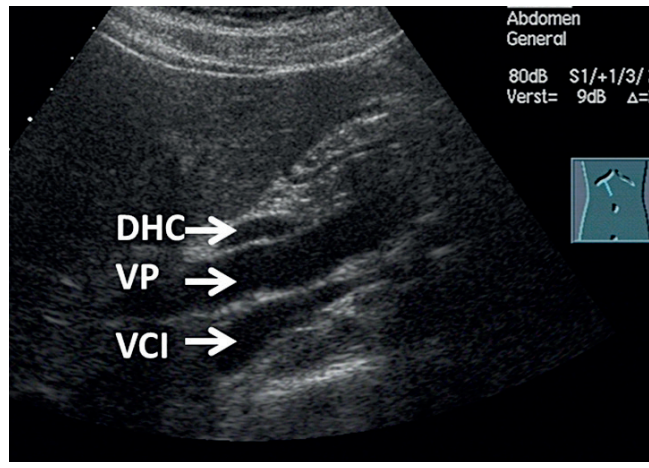

Abb. 4 Drei hintereinander liegende echofreie tubuläre Strukturen im Schulter-Nabel-Schnitt: In der Tiefe die Vena cava inferior ( $\mathrm{VCl}$ ), darüber die Vena portae (VC) und wiederum ventral davon der hier kräftige Ductus hepatocholedochus (DHC).

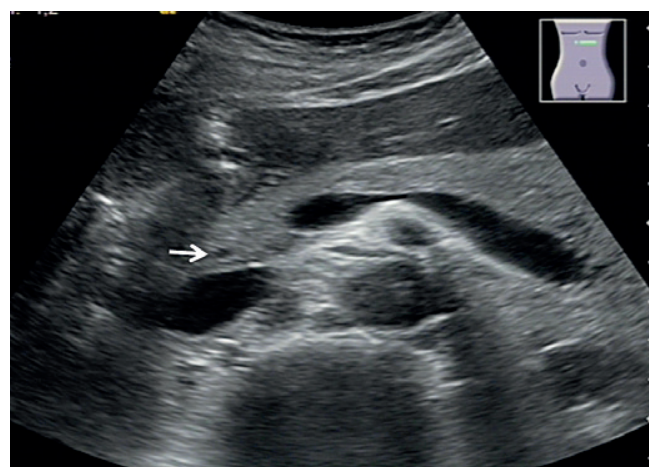

Abb. 6 Im Oberbauchquerschnitt ist im Pankreaskopf ventral der $\mathrm{VCl}$ der quergeschnittene $\mathrm{DHC}$ als winziger echofreier Kreis (Pfeil) zu erkennen.

Wie schmal ist normal? | Bei jungen gesunden Menschen kann der DHC sehr schmal $(<2 \mathrm{~mm})$ sein ( Abb. 7). Als Faustregel kann immer noch gelten, dass ein Durchmesser $<7 \mathrm{~mm}$ nicht auffällig ist. Ob der DHC im Alter und nach Cholecystektomie weiter wird oder nicht, wird gleichfalls noch debattiert [2,3].

Cave Auch ein schmaler DHC kann Steine tragen und ein weiter eine Normvariante sein. Bei diskordanten Befunden: neu nachdenken!

Ganz wichtig ist, sonografische Befunde immer im Zusammenhang mit der Klinik und anderen (Labor-) Befunden zu interpretieren.

\section{Wenn Unsicherheiten bleiben}

Erneute Untersuchung I Ließ sich der DHC im Rahmen der Abdomensonografie aufgrund von schlechten Schallbedingungen nicht gut darstellen, kann man den Patienten am nächsten Morgen nüchtern erneut untersuchen.

Keine Darstellung - keine Dilatation I Ist der DHC auch bei subtiler Untersuchung nicht darstellbar und sind alle tubulären Strukturen in der Leberpforte in der Farbdopplersonografie farbbelegt,

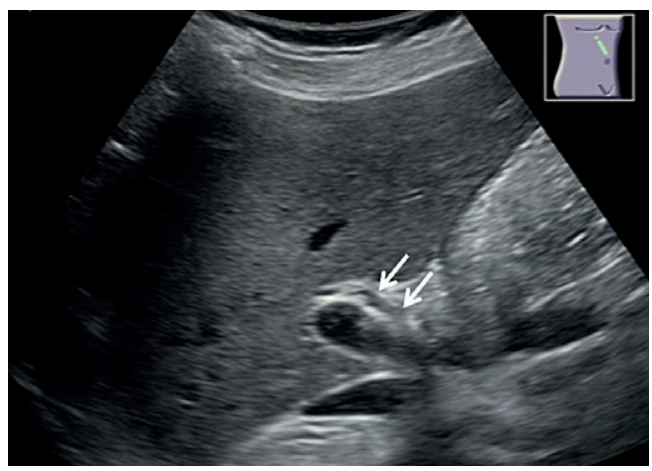

Abb. 5 In Linksseitenlage kann trotz vollen Magens (links im Bild) der DHC (Pfeile) gut dargestellt werden.

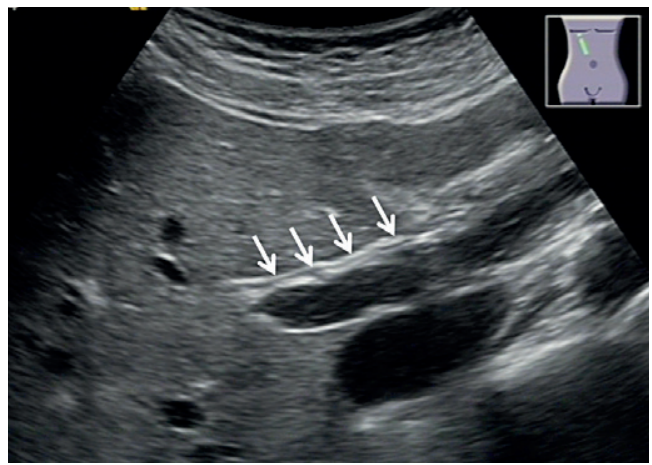

Abb. 7 Der DHC (Pfeile) eines gesunden Probanden, dargestellt im Schulter-Nabel-Schnitt, mit einem Durchmesser von $1,8 \mathrm{~mm}$.

kann man immerhin indirekt eine Dilatation des DHC ausschließen.

Bei Zweifeln: Endosonografie | Bleibt die Darstellung lückenhaft, aber der Verdacht auf eine Dilatation oder Steine bestehen, ist die Endosonografie die Methode der Wahl, um den Gallengang weiter zu untersuchen. Mit zunehmender Erfahrung werde Sie jedoch schnell immer mehr Diagnosen „perkutan“ stellen können.

\section{Wichtiges in Kürze}

- Die Pfortader ist die dorsale Leitstruktur zum Auffinden des DHC.

- Deutlich sichtbare Gallenwege neben den Pfortaderästen in der Peripherie sind ein Hinweis auf einen gestörten Gallenabfluss.

- Pfortader und Arteria hepatica propria können mit dem DHC verwechselt werden.

- Die Linksseitenlage kann sehr nützlich sein.

- Der gesunde DHC hat eine erkennbare Wand, die nicht geschichtet, sondern stark echogen ist.

- Befunde immer in Zusammenhang mit Klinik und Labor interpretieren.

Literatur:

1 Schmidt G, Görg C. Kursbuch Ultraschall. Thieme, Stuttgart 2015

Vollständiges Literaturverzeichnis unter http://dx.doi.org/10.1055/s-0041-101383

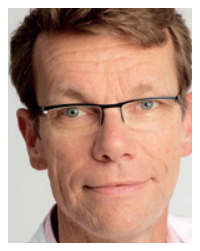

Dr. med. Thomas Müller ist Gastroenterologe und DEGUM-Kursleiter Innere Medizin. Er arbeitet als Oberarzt in der Medizinischen Klinik I am Klinikum am Steinenberg in Reutlingen. mueller_tho@klin-rt.de

Interessenkonflikt Dr. Thomas Müller erhielt Reisekostenunterstützung bzw. Vortragshonorare der Firmen Esaote Biomedica, Hitachi Medical Systems und Toshiba.

DOI 10.1055/s-0041-101383 Dtsch Med Wochenschr 2015; 140: 587-589

(c) Georg Thieme Verlag KG . Stuttgart · New York . ISSN 0012-0472 
2 Sienz M, Ignee A, Dietrich CF. Normwerte in der Abdomensonografie - biliopankreatisches System und Milz. Z Gastroenterol 2011; 49: 845-870

3 Valkovic P, Miletic D, Zelic M, Brkljacic B. Dynamic Changes in the Common Bile Duct After Laparoscopic Cholecystectomy: a Prospective Longitudinal Sonographic Study. Ultraschall Med 2011; 32: 479-484 